\title{
Editorial - L'idéologie de la menace du Sud
}

Didier Bigo

\section{(2) OpenEdition}

\section{Journals}

\section{Édition électronique}

URL : http://journals.openedition.org/conflits/2012

DOI : $10.4000 /$ conflits.2012

ISSN : $1777-5345$

Éditeur :

CCLS - Centre d'études sur les conflits lilberté et sécurité, L'Harmattan

Édition imprimée

Date de publication : 17 mai 1991

ISSN : 1157-996X

Référence électronique

Didier Bigo, «Editorial - L'idéologie de la menace du Sud », Cultures \& Conflits [En ligne], 02 | printemps 1991, mis en ligne le 31 mars 2006, consulté le 30 mars 2021. URL : http://journals.openedition.org/ conflits/2012; DOI : https://doi.org/10.4000/conflits.2012

Ce document a été généré automatiquement le 30 mars 2021.

Creative Commons License 


\title{
Editorial - L'idéologie de la menace du Sud
}

\author{
Didier Bigo
}

1 Actuellement se construit un discours sur la ou les menaces du Sud qui envahit les médias, touche les hommes politiques des différents pays et structure en grande partie les réactions de l'opinion à la guerre du Golfe. Pourtant ce discours passe relativement inaperçu et presque personne ne cherche à en décrypter l'idéologie sous-jacente alors qu'elle est sans doute une de ces nouvelles lignes de clivages qui traversent de manière flagrante à la fois les analyses des relations internationales et celles des différents partis politiques, poussant ceux-ci à des recompositions douloureuses.

2 Ce numéro deux de Cultures \& Conflits a pour objet d'étudier ce discours, de voir sur quoi il repose et les restructurations de l'espace politique qu'il implique. Nous espérons que le débat que nous ouvrons sur ce thème sera prolongé et que les médias, tout comme la société politique et la société civile, prendront conscience de son importance. En effet, ce discours sur la "menace du Sud" en recomposant la scène internationale et les jeux politiques internes, en se nourrissant des succès au Maghreb de l'idéologie jumelle sur la menace du Nord, a un impact et des conséquences qui seront lourdes pour l'avenir. Ces discours alimentent des peurs, des fantasmes qui fabriquent des deux côtés un sentiment d'angoisse et de méfiance pouvant se transformer en franche hostilité et en discours de haine (anti-occidentalisme, racisme...). De plus, ils ont tout pour devenir, de par leur croisement, une prophétie auto-réalisatrice. C'est pourquoi il est urgent de s'attaquer immédiatement à leurs racines pour en montrer les faiblesses, les incohérences, et les logiques de fonctionnement. Le numéro traitera essentiellement de celle qui nous concerne directement, l'idéologie de la menace du Sud mais la similitude de fonctionnement et l'insécabilité de la relation entre idéologie de la menace du Sud en Europe et aux Etats-Unis, et l'idéologie de la menace du Nord contre les valeurs du monde arabe au Maghreb et au Proche-Orient, permettent de mieux comprendre pourquoi certains fabriquent artificiellement un conflit de cultures en opposant celles-ci de manière irrémédiable. 
3 D'où une première question : le discours sur la menace du Sud s'appuie-t-il sur un argumentaire réaliste et des risques fondés (démographie, immigration, terrorisme, prolifération d'armements...) ou s'agit-il d'une construction idéologique qui, à la fois remplit des fonctions essentielles dans la redéfinition des identités (nationales, religieuses, politiques...) et dans les perceptions croisées des sociétés et des cultures, c'est-à-dire dans la manière dont on se représente et dont on envisage le rapport à l'Autre'

4 A l'analyse, il ne fait guère de doute que la deuxième hypothèse est la bonne : il ne s'agit pas d'une appréciation réaliste de risques objectifs à l'échelon international ou national, mais d'une idéologie de la menace s'alimentant aux fantasmes de nos sociétés et puisant en eux de quoi entretenir une peur, un sentiment d'insécurité disproportionnés et déconnectés de toute réalité. Ainsi, aux yeux de tous ceux qui ont participé à ce numéro, l'essentiel est-il de décrypter cette idéologie et d'en montrer les incohérences, mais on verra qu'en revanche il n'existe pas forcément d'unanimité entre les auteurs sur les multiples dimensions que revêt cette idéologie et sur sa valeur de "vérité".

5 Refuser les simplifications, les métaphores qui les orientent et créent des hiérarchisations pour le moins contestables en inventant d'autres représentations, d'autres lectures du monde contemporain, devient alors fondamental et dans la prolongation des hypothèses de notre premier numéro sur l'autonomisation des conflits, de la diversification de leurs trajectoires, il nous semble que l'on peut opposer à ces lectures pauvrement binaires, en terme de rapport Nord/Sud (que ce soit sous les catégories de l'économique, du politique ou du culturel), des lectures plurielles qui insisteront au contraire sur la diversité et l'irréductibilité absolue des réalités sociopolitiques contemporaines mondiales ${ }^{2}$.

6 Louis-Jean Duclos démontre, en autre, l'ambiguïté des notions de Nord et de Sud dont la cohérence est artificielle, et dont la présentation manichéenne occulte le véritable fonctionnement des relations internationales contemporaines. Mais il semble aussi que ces termes de Nord et Sud reviennent malgré tout "spontanément" dans les analyses comme s'il était quasi impossible de penser la pluralité du monde contemporain, la diversité des trajectoires historiques, et comme s'il était nécessaire d'avoir un terme générique pour subsumer certaines catégories économico-politiques maintenant que la notion de tiers-monde est désuète. Les identifications de certains continents, de certains pays, de certains régimes politiques au Sud et d'autres au Nord comme s'il existait une coupure objective entre deux mondes, deux cultures sont pour le moins illusoires (où placer l'Afrique du Sud, Singapour, la Corée, etc.?) même si la marginalisation économico-stratégique de certaines zones comme l'Afrique subsaharienne ou l'Amérique andine et l'approfondissement des inégalités de développement mondial sont bien réelles dans le domaine de l'économique.

7 En effet les processus économiques et politiques à l'œuvre créent des fractures irrémédiables entre les anciens pays dits du Sud ou non alignés ou tiers-monde et créent en même temps des interdépendances telles entre certains pays dits du Nord et d'autres dits du Sud que les chevauchements en terme d'économie, de mouvements de populations, d'échanges culturels contredisent tous les jours l'image de la coupure.

8 Ces lectures plurielles montrant l'inanité des réductions binaires, nous allons les retrouver tout au long du numéro. Elles s'attaquent aux racines mêmes de ces discours et de leurs pseudo-évidences. En effet, en étudiant de près les soi-disant problèmes de 
sécurité posés par l'immigration et les populations dites "musulmanes" (c'est-à-dire assignées d'office à une religiosité qui est loin de déterminer leur identité) installées depuis plusieurs générations sur le territoire européen, on s'aperçoit à quel point le débat est biaisé car, non seulement les réponses sont mauvaises, mais les questions sont surtout mal posées et souvent relayées telles quelles par les médias(3).

Interprétations à l'emporte pièce sur les structures démographiques comparées des pays, sur les taux de fécondité, sur les mécanismes de transition démographique, sur les liens entre démographie, structure par âge et économie dont Mustapha Sehimi nous donne les principales clés d'analyse...

Confusions entre terrorisme, grande criminalité, immigration et réfugiés, non seulement de la part des médias mais aussi de certaines bureaucraties nationales et européenne, remettant en partie en cause le droit d'asile et une tradition d'ouverture indispensable, à notre avis, pour que l'on puisse parler d'espace démocratique... Interprétations caricaturales sur les liens entre les pays d'origine, les idéologies de leur gouvernants ou de mouvements d'opposition et les attitudes des populations immigrées en Europe, discours sur l'intégrisme sans identification des locuteurs et des groupes sociaux qui permet des amalgames pour le moins ambigu sur les associations islamiques en France que l'on suppose bien rapidement être les relais des idéologies de leur pays d'origine ainsi qu'une série de discours douteux sur le "retour du religieux"... Remise en cause du bienfait du multipartisme et des élections démocratiques lorsqu'elles risquent de porter au pouvoir des individus dont les valeurs sont différentes de l'Occident.

Il en va de même, comme le montrent Pierre Dabezies, Marie-Lucy Dumas et Bertrand Warusfel, pour la prolifération balistique, nucléaire et chimique, dont on peut certes s'inquiéter, mais dont le rapport avec les menaces du Sud contre le Nord est pour le moins évanescent et hautement hypothétique étant donné les rapports de force présents. En effet, si le débat à un sens, il doit être replacé dans le cadre des ambitions hégémoniques régionales de quelques pays autant du Nord que du Sud (Israël, Afrique du Sud, Corée, Inde, Pakistan, Irak...) à l'égard de leur environnement immédiat et dans celui de la légitimité pour un "Club" de puissances de disposer d'armements que l'on interdit aux autres Etats.

Le débat sur les "risques sociétaux" (terme euphémisé utilisé par certains stratèges pour éviter celui de menace) est aussi particulièrement biaisé. On reproche avant tout aux pays les plus pauvres, leur pauvreté, leur manque de malthusianisme démographique, leurs conditions sanitaires, leurs conflits internes... et l'on en fait des "risques exportables vers les pays développés" ! Ces fausses menaces dont nous parle Christiane Hurtig à propos de l'Inde sont pourtant souvent évoquées. Or, cette partie du discours plus insidieuse, moins marquée par ses origines, se développe de plus en plus. Ainsi un certain discours "humanitaire" succombe lui aussi à la prégnance de cette idéologie et justifie le maintien de l'aide, non par la nécessité du développement des pays africains, latino-américains et asiatiques, mais pour éviter que la marginalisation du continent africain par exemple ne soit une bombe à retardement dans les rapports Nord/Sud et ne débouche soit sur une désagrégation sociale épidémique finissant par s'infiltrer au Nord avec les réfugiés que cela produirait, soit sur l'affrontement des deux blocs Nord/Sud. Il en va de même du discours pourtant a priori antagoniste avec cette idéologie, celui sur la prochaine libre circulation pour tous les ressortissants à l'intérieur des frontières européennes (acte unique de 1985) qui se constitue à travers 
l'élaboration de mesures compensatoires (accords de Schengen, conférence Trevi) qui renforceront les contrôles des frontières externes et sont perçus de facto comme une fermeture de l'Europe à l'immigration, comme la mise en place d'une Europe frileuse retranchée derrière ses privilèges de nantis et guère disposée à en partager les privilèges avec un monde maghrébin à ses portes ${ }^{3}$.

Les racines de l'idéologie de la menace du Sud

D'où vient alors la force de conviction de cette idéologie de la menace du Sud, si elle n'est pas liée à une quelconque valeur de "vérité" ? N'est-ce pas un discours instrumentalisé par certains groupes sociaux, par des intérêts corporatistes et par des groupes politiques qui, pour des raisons diverses, se trouvant privés d'un discours traditionnel et bien rôdé depuis la fin de la bipolarité Est/Ouest, retrouvent avec l'idée d'antagonisme Nord/Sud un biais qui leur permet au moindre coût de reprendre leur argumentaire habituel ? En effet on passe d'une bipolarité à une autre ce qui permet d'user d'une matrice quasi identique même si cela produit quelques effets de brouillages significatifs comme le montre la position "pacifiste" lors de la guerre du Golfe d'un Parti communiste mais aussi d'un Front national et de certains hauts responsables militaires peu habitués à se voir cohabiter sur la même ligne. A ce propos Denis Duclos, dans un texte très suggestif, montre à quel point le basculement d'une métaphore (Est/Ouest) à une autre (Nord/Sud) n'est pas sans conséquence sur les visions du monde et sur les identités que l'on se construit à propos de Soi et de l'Autre. Identifier plus précisément les locuteurs de ces discours, leur contenu précis, les amalgames qu'ils fabriquent semble alors une priorité de l'analyse afin de ne pas succomber au charme facile de construire une idéologie inverse de la première mais tout aussi éloignée des réalités. John Kenneth Galbraith, Michael Klare aux Etats-Unis, Maurice Bertrand en France ont ouvert des pistes et ont montré le rôle clé des lobbies des industriels de l'armement et de certains "think tank" du Pentagone ainsi que la nécessité pour eux d'instrumentaliser un discours sur la menace du Sud, en lui donnant une cohérence et un impact médiatique que ce discours n'avait pas jusqu'alors. Une généalogie rapide des strates de cette idéologie, suivant cette piste de recherche, n'est pas alors sans intérêt. Ce discours sur la menace du Sud comme substitut à la menace de l'Est a pris racine bien avant la fin de cette dernière. Les théories sur les conflits de basse intensité du Pentagone contre les "subversions latino-américaines" en furent sans doute les premiers éléments mais, en tant que tel, on peut dire que c'est avec l'interprétation de la révolution iranienne en terme de radicalisme islamique à vocation prosélyte et déstabilisante pour l'Occident qu'il s'est structuré. Il a pris son essor à ce moment et, au delà de l'Amérique latine, a englobé le Proche et le MoyenOrient pour les Américains ${ }^{4}$. Parallèlement, les différents pays européens, plus ou moins touchés par les retombées des conflits au Moyen-Orient, ont ajouté leurs propres peurs à l'égard de l'Afrique et du Maghreb en particulier, à la construction de cet agrégat d'un Sud menaçant. De plus la montée de "puissances régionales" a été perçue comme un risque de remise en cause de la stabilité mondiale par les stratèges américains. Mais cette explication est en elle-même insuffisante. Au delà d'une telle instrumentalisation très spécifique, nous avons essayé de montrer ailleurs que l'impact et la popularité de ce discours sur la nouvelle menace tiennent plus profondément à la causalité diabolique qu'il remplit et à la restructuration d'identité qu'il permet face à une nouvelle figure de l'ennemi ${ }^{5}$. Si aujourd'hui nous parlons donc d'idéologie de la menace du Sud c'est pour signifier que ces lectures qui ramènent la réalité sociale internationale à un discours de type affrontement Nord/Sud, non seulement servent 
des intérêts, mais restructurent nos visions du monde et fabriquent un système clos sur lui-même qui a comme prémisse d'énoncer que tout nouvel événement international peut s'expliquer à partir de cette idée initiale : le nouvel ennemi est à chercher au Sud.

Selon l'implacable logique réductionniste qui caractérise ces idées, il est nécessaire de penser le monde de manière binaire en identifiant qui appartient à quoi, et surtout il faut agir le plus rapidement possible afin que la dissuasion du fort au faible maintienne un rapport de force favorable en faveur du Nord : rapport de force qui, sinon, à long terme se renverserait en sa défaveur. Ceci n'est d'ailleurs pas sans rappeler la manière dont un système totalitaire pense le monde en jouant des angoisses de l'avenir, des catégories oniriques mais mobilisatrices de races ou de classes en enfermant la réalité dans une grille de lecture qui a comme vocation de tout expliquer ${ }^{6}$.

Ainsi l'idéologie de la menace du Sud en Occident, tout comme son miroir au MoyenOrient : l'idéologie anti-occidentale, procède de manière manichéenne en diabolisant l'adversaire et en se purifiant, en s'idéalisant dans le mouvement même de la dénégation de l'Autre. Elle fabrique, comme le souligne Gabel, une illusion de centralité qui permet à l'individu ou aux groupes qui y croient, de supprimer les doutes, le malaise de n'être plus des sujets historiques centraux dans une histoire en mouvement ${ }^{7}$. Elle permet, croit-on, de lutter contre cette "mélancolie démocratique" qui a saisi l'Ouest depuis que le communisme s'est effondré, depuis que le frère ennemi est décédé brutalement ${ }^{8}$. Elle reforge une identité "nationale" "européenne", "occidentale" qui était en perdition et se restructure par la création d'une menace synthétique du Sud (tout continent confondu) provenant de leurs États (prolifération balistique, terrorisme), mais aussi de leurs sociétés (démographie, pauvreté, épidémie de sida, culture de plantes servant de base à des substances hallucinogènes) et de leurs individus (immigration, réfugiés...). Menace multiforme dont le mixte, plus ou moins homogène, renforce justement la dangerosité puisque, transnationale, la menace ou les menaces échappent à une parade militaire classique et obligent à une nouvelle conceptualisation de la sécurité, plus globale, plus totale et imbriquant profondément sécurité extérieure et sécurité intérieure.

Comme dans un délire paranoïaque, tout vient s'agencer dans ce discours, et tout élément contradictoire à la thèse est lu sur le mode du complot et de la ruse ${ }^{9}$. La résurgence de thèmes racistes et d'une idéologie d'extrême droite ne doit pas alors nous surprendre. La menace du Sud en est en quelque sorte le prolongement sur le plan international. Néanmoins, avant l'effondrement du système communiste, ce discours restait confiné à des cénacles militaro-industriel limités, à des groupes à l'idéologie extrême-droitière et il était perçu comme l'expression très marginale d'angoisses à l'égard d'un "colonialisme à l'envers" qui faisaient sourire ou qui étaient traitées par le mépris.

La nouveauté tient sans doute à ce que depuis la chute du mur de Berlin, ce discours s'est affranchi de cette origine qui le marginalisait, pour devenir un discours général, partagé par de nombreuses personnes dont les repères identitaires étaient brouillés (fin de la coupure nette entre Est et Ouest, construction européenne...), et qui ont eu tendance à s'accrocher à cette idée d'une nouvelle menace venue du Sud comme à une bouée de sauvetage. Cette idéologie de la menace du Sud est venue s'insérer dans les déconvenues de l'idéologie de la paix et de la démocratie de certains intellectuels qui avaient confondu la fin de l'antagonisme Est/Ouest en Europe avec la pacification de la planète, des auteurs de gauche comme Régis Debray dans "tous azimuts" l'ont sans 
doute généralisé, et le gouvernement français lui-même, par l'intermédiaire de son ministre de la Défense, a participé à cette propagation créant un climat particulièrement favorable à l'incompréhension réciproque des acteurs lors de la crise du Golfe ${ }^{10}$.

Crise et guerre du Golfe à la lueur de l'idéologie de la menace du Sud.

Cette crise, qui se caractérise sans doute par la forte altération du sens des messages entre les adversaires et leur certitude pourtant de s'être fait comprendre, ne s'explique en effet que par la prégnance à Washington, Londres et Paris de ce discours sur les nouveaux adversaires de l'OTAN(${ }^{(1)}$, et à Bagdad, Alger et autres capitales du monde arabe, sur le discours miroir de l'anti-occidentalisme, des ennemis de l'Islam et de l'Oumma. Mais, avant l'action armée, on peut supposer qu'elle ne dépassait, dans un cas comme dans l'autre, que très peu les cercles dirigeants et certains groupuscules. En revanche, avec cette dernière, des groupes sociaux entiers se sont vus mobilisés et ont répondu positivement aux machines de propagande.

Ainsi, la guerre du Golfe a renforcé et modifié cette appréhension biaisée des réalités des pays du Sud, elle a refabriqué imaginairement pour beaucoup d'acteurs et d'interprètes deux camps, opposés de manière manichéenne, et se résumant à une opposition Nord/Sud, où le Nord serait incarné par Georges Bush et le Sud par Saddam Hussein. Une bonne partie des réactions à la guerre du Golfe et à sa prolongation actuelle reste marquée par cette interprétation d'un antagonisme global Nord/Sud.

Or, ces discours, très nombreux, quand bien même ils seraient critiques vis-à-vis du "vainqueur", ce qui est le cas dans ce numéro des articles de Bernard Ravenel ou de Sergio Vieira, risquent peut être, à partir du moment où ils reprennent cette problématique globalisante, de rester dans celte représentation Nord/Sud (dont ils critiquent à juste titre les effets) au lieu de la faire éclater afin de montrer la multiplicité du Sud et du Nord ainsi que les chevauchements permanents entre ces deux ensembles flous. II me semble alors que malgré le conflit du Golfe, en dépit de lui, il n'y a toujours pas deux camps opposés à l'échelle mondiale qui se reconnaitraient par leur appartenance à des valeurs différentes et antagonistes mais la table ronde animée que nous avons organisée a montré qu'il y a là un débat encore très vif

Si la guerre du Golfe ou plus exactement, car la précision des termes est utile pour délimiter les séquences temporelles évoquées, la guerre entre l'Irak et la coalition internationale sous direction américaine a produit un tel impact dans l'imaginaire, elle ne le doit donc pas uniquement au différentiel technologique et à la rapidité d'un conflit international que nombre d'analystes et d'hommes politiques prévoyaient long et sanglant, elle ne le doit pas non plus exclusivement à ce qu'elle fut une guerre télévisuelle suivie par tous, elle le doit avant tout au fait qu'elle s'est moulée dans ce discours sur la menace qui lui préexistait et qu'elle n'a fait que renforcer en le polarisant et en le "réel(isant)"12. Elle le doit donc à des systèmes de représentations dont l'antagonisme de contenu (Saddam/Bush, Irak/USA, monde arabe/coalition internationale, monde musulman/occident, déshérités/nantis, dictature/démocratie, Sud/Nord...) ne cache pas les similitudes formelles et la dimension mimétique : stigmatisation de l'adversaire, utilisation d'un répertoire quasi-religieux à des fins d'homogénéisation d'un bloc contre un autre, et d'allégeance sans condition au leader du bloc, tentative de mobilisation concentrique d'identités toujours plus éloignées du leader mais toujours plus globalisantes et mondiales... 

de leur population, d'autres ont cru, au nom de leurs propres valeurs, légitime de soutenir un dictateur arabe parce qu'il était arabe contre "l'agression" occidentale et ce, au nom des masses qui se seraient retrouvées derrière Saddam Hussein. Beaucoup ont parlé au nom de ces groupes sociaux divers du monde arabe à qui l'on a prêté toutes les positions que l'on voulait prôner. On en a rajouté de part et d'autre sur l'altérité culturelle, sur le faux universalisme occidental masquant ces intérêts... et tout ceci n'est pas faux. Seulement hypostasier les différences, fabriquer des coupures radicales, mettre en place un apartheid culturel, c'est participer, sans le savoir, au développement de cette idéologie de la menace qui diabolise l'adversaire. Une fois de plus les mécanismes mêmes de mobilisation, les techniques de propagandes, l'absence d'embrasement généralisé sur lequel on a fantasmé (terrorisme, cinquième colonne, assassinat d'étrangers aux pays, guerre populaire à l'échelle mondiale) montrent au contraire une identité de fait des adversaires et leur appartenance à un même monde, que cela leur plaise ou non. mais non de sombrer dans un culturalisme lui assignant une nature dont il ne pourrait se défaire. Il n'y a pas d'inéluctabilité de la prégnance du religieux dans le monde arabe qui condamnerait d'avance la possibilité d'une autonomisation d'un espace public, pas plus qu'il n'est impossible d'y promouvoir des discours sur les droits de l'homme et la laïcité . Il n'y a pas non plus un échec forcé de la modernisation par inadéquation des valeurs et inaptitude à la comprendre ${ }^{14}$. Il faut peut-être arrêter ces discours pour le moins ambigu sur les "frustrations des masses arabes" à l'égard de l'Occident,, de la modernité, de sa richesse... et éviter le thème récurent d'une humiliation du monde arabe qui aurait une revanche à prendre (sur l'Occident ou sur lui-même...). consistance, sont pour le moins sommaires. Elles se résument souvent à quelques affirmations péremptoires selon lesquelles les masses arabes en manifestant leur 
soutien à Saddam Hussein auraient montré leur réelle frustration contre l'Occident. Affirmations péremptoires car le moment manifestant pour reprendre l'expression de Pierre Favre, s'il donne une indication de la capacité à mobiliser d'une organisation ne décrit en rien l'état de l'opinion de l'ensemble de la population. De plus la thèse de l'explication du passage à la mobilisation et à la violence par la frustration ou la frustration relative n'a guère convaincu, faut-il le rappeler, la communauté scientifique. Charles Tilly a au contraire montré comment les répertoires de violence répondaient à des stratégies d'acteurs rationnels, contraints par la structuration de leur champ politique et leur mémoire historique. Alors, oubli de cette réflexion dès qu'il s'agit du monde arabe ? Croyance en l'inaptitude de ce dernier à instrumentaliser la violence et à en user rationnellement ?

"Privilège" de l'irrationnel ? Si, véritablement, on veut comprendre les mobilisations liées à la guerre du Golfe, commençons par leur appliquer les mêmes grilles de lecture que partout ailleurs, au lieu de se laisser égarer par les fantasmes culturalistes que nous entretenons à son égard ${ }^{15}$.

Ce numéro n'est donc pas, on l'a compris, un numéro de plus sur le conflit du Golfe, même si celui-ci s'inscrit dans le thème central que nous traitons. L'idéologie de la menace du Sud ne se réduit pas à l'enjeu de la guerre du Golfe. Elle touche à l'ensemble des perspectives géostratégiques mondiales, à la discussion sur la prolifération balistique, chimique et nucléaire, mais aussi aux crises sociétales de certains pays que J.-C. Rufin désignent sous le terme de "limes". Elle détermine la nouvelle vision du monde que l'on se fait depuis la fin de la bipolarité.

Espérons qu'à la lecture de ce numéro, cette idéologie qui s'infiltre dans de nombreux secteurs, soit perçue pour ce qu'elle est : un discours d'exclusion, un discours de combat ne reposant sur aucune demi-vérité mais sur des perceptions fantasmatiques de la réalité.

\section{NOTES}

1.par exemple l'image d'une civilisation méditerranéenne ou au contraire d'un antagonisme islam/chrétienté autour de la Méditerranée.

2.D'autant que l'on notera le glissement significatif des discours du dialogue Nord/Sud à la menace du Sud sur le Nord.

3.Allusion à la manière dont Jean-Marie Le Pen avait réussit à imposer ses thèmes à la classe politique française : de mauvaises réponses à de bonnes questions.

4.Michael Klare, Low Intensity Warfare, Pantheon Books, New York, 1989.

5.Didier Bigo, Daniel Hermant, "Les lectures de la conflictualité", Stratégique, $n^{\circ} 47$.

6.Hannah Arendt, Le système totalitaire, Gallimard, Paris, 1972.

7.Gabel, L'idéologie, Encyclopedia Universalis, 1980.

8.Pascal Bruckner, La mélancolie démocratique, Paris, Seuil, 1990. 
9.D'où chez certains un engouement pour l'analyse de la "taquiah", la ruse qui permet aux chi'ites de ne pas être tenus moralement de dire la vérité à un infidèle et qui devient dans ce discours le comble de la perversité, la preuve de leur mauvaise foi... 10.Pour des développements sur ce thème voir Didier Bigo, "Les interprétations des années 1989/1990", Approches Polémologiques, IFP/FEDN, 1991.

11.Les futurs anti-missiles français présentés au salon du Bourget vont parait-il être déployés le long des côtes méditerranéennes.

12.On notera d'ailleurs que cette séquence temporelle a tendance à occulter chez certains la phase précédente du conflit local où l'Irak a envahi le Koweït, et chez les autres ou chez les mêmes la phase actuelle où le conflit est devenu une guerre civile sous ingérence humanitaire entre le régime irakien, les Kurdes et les chi'ites irakiens. Elle concentre ainsi les effets de réel et refabrique une idée d'une guerre courte, propre...recréant artificiellement une rationalité de la guerre a posteriori.

13. Voir l'appel d'Esprit à une pétition contre la guerre des cultures que nous relayons dans ce numéro.

14.A l'inverse, le monde occidental n'est pas sans valeurs, purement dominé par la technique et l'argent. Il a ses propres valeurs locales.

15.Dans les numéros 5 et 6 de Cultures \& Conflits consacrés aux contestations populaires et aux violences urbaines, nous reviendrons sans doute sur ce thème.

INDEX

Mots-clés : (in)sécurité, discours sécuritaire, menace, pauvreté

Index géographique : Pays du Sud (Tiers Monde), Relations Nord/ Sud

Index chronologique : post-guerre froide 